\title{
Green Nanotechnology
}

\author{
Geoff B. Smith \\ School of Physics and Advanced Materials, University of Technology, Sydney \\ Po Box 123, Broadway, NSW, 2007 Australia
}

\begin{abstract}
Nanotechnology, in particular nanophotonics, is proving essential to achieving green outcomes of sustainability and renewable energy at the scales needed. Coatings, composites and polymeric structures used in windows, roof and wall coatings, energy storage, insulation and other components in energy efficient buildings will increasingly involve nanostructure, as will solar cells. Nanostructures have the potential to revolutionize thermoelectric power and may one day provide efficient refrigerant free cooling.

Nanomaterials enable optimization of optical, opto-electrical and thermal responses to this urgent task. Optical harmonization of material responses to environmental energy flows involves (i) large changes in spectral response over limited wavelength bands (ii) tailoring to environmental dynamics. The latter includes engineering angle of incidence dependencies and switchable (or chromogenic) responses. Nanomaterials can be made at sufficient scale and low enough cost to be both economic and to have a high impact on a short time scale. Issues to be addressed include human safety and property changes induced during manufacture, handling and outdoor use. Unexpected bonuses have arisen in this work, for example the savings and environmental benefits of cool roofs extend beyond the more obvious benefit of reduced heat flows from the roof into the building.
\end{abstract}

Keywords: energy efficiency, windows, cool roofs, paints, nano-coatings, spectral selectivity, angular selectivity, chromogenics

\section{INTRODUCTION}

The terms "Greentech" and "Cleantech" have become part of everyday language. Given their growing use as a marketing tool for products and science proposals, a clearer notion of what they encompass is worth formulating. Then the reasons nanoscience and in particular nanophotonics and thin films are so important becomes clear. My experience is the scientific community, and the population at large have only vague notions of what the modern use of the terms "Green" or "Clean" actually means in the context of technology and science? As for quantifying "greenness" for any particular technology, though improving its elements are still poorly understood, but very important and illuminating [1]. The words energy, environment, pollution, sustainability, global warming, and resources, all come up in qualitative analyses, but relevant numbers are crucial yet all too often not provided. Underpinning all of these is the link between the quality of human existence and the thermodynamic and electromagnetic properties of the environment that supports it. This link has been weakening over the past 250 years. The quality of all life in future will hinge on creating something new, an over-arching harmony between technology and nature. This paper explores some of the likely elements involved in this task.

email g.smith@uts.edu.au

Nanostructured Thin Films IV, edited by Raúl J. Martín-Palma, Yi-Jun Jen, Tom G. Mackay,

Proc. of SPIE Vol. 8104, 810402 · (c) 2011 SPIE · CCC code: 0277-786X/11/\$18 · doi: 10.1117/12.893114

Proc. of SPIE Vol. $8104810402-1$ 
For a "green" nanoscientist or engineer stepping back to consider these broader questions in scientific terms I believe can lead to much better focus within particular projects, hence higher productivity, less wasted empirical effort, and the ability to better "mine" and use for energy and environmental related applications the explosive emerging array of new fundamental developments in optical, electrical, thermal, chemical and mechanical nanoscience. The urgency of de-carbonising our environmental impact and remediating past damage, while satisfying the needs of a growing population, and lifting the quality of life for many of these, requires a new breed of scientists for which a useful distinguishing label might be "new-green". They are not traditional environmental scientists, who focus on bio-eco-systems and chemistry. The latter however will increasingly need "new-green" input, while this new breed will use key elements of natural science including bio-mimickry, plus greater emphasis on the physical aspects of nature including, as will be demonstrated later in this paper, global and local climate science. They will rely on a growing army of traditional chemists, physicists, agricultural and environmental scientists, whose work must also advance, and be guided to some extent by the "new-green" paradigm. While some architects and engineers already fit the "new-green" criteria, a much wider understanding of these issues is now needed among our design decision makers.

The scope of the emerging needs also means that economic aspects of any new technology must be considered. The world needs new products and improved versions of established products at commodity scales and low cost, to be met comfortably with existing resources. Lowering cost is not only about resources but also includes the energy and time used in manufacturing, plus supply and installation issues. Production scale capabilities are one of the core attractions for this task of select nano- and optical technology, as we shall elaborate. My group's applied nano-photonic research in this field has been guided by this "new-green" principle. It relies on and also stimulates, new basic science. Examples follow.

Many human needs come into the "green" equation and energy is just one aspect. The image of "green" is changing but past perceptions encompassing ugly, unwieldy structures and uncomfortable lifestyles, along with many failures to live up to expectations persist. Past association with a focus on nature, excluding economics, human needs and political reality, is fading. Advanced economies expect quality lifestyles, not turning back the clock. The aim is thus "green" combined with attractive, reliable and durable. This is core to our "new harmony". The "old”, often romaniticised pre-technological harmony cannot support earth's billions. Two aspects of the traditional green movement, getting closer to nature and better preserving nature, plus reducing city pollution, are retained as they will be integral to our universal goals. As will be detailed in section 3, making the most of what the environment has to offer without hurting it will put all of us in closer touch with the natural environment, which is uplifting and dynamic, leading to health and productivity benefits. Developments in the $20^{\text {th }}$ century, in particular air-conditioning, the fluorescent tube, and the internal combustion and jet engines, meant many spent most daytime hours in sterile and polluted environments away from links to the natural world in which we had evolved. Our "new-green" nanotechnology aims to restore that link, without destroying the benefits we associate with good lighting, thermal comfort, reliable transport, and so on. There are bonus novel benefits in this "new-green" world, which need more study and are sadly as yet mostly not appreciated. A wider grasp of the value these add would accelerate progress.

Technology today is about provision of human needs efficiently and in high volumes. These include food, potable and irrigation water, processed materials, health, energy, light, transport, durable shelter, thermally comfortable buildings and clothing, quality air, tools and machines, education, communication, entertainment, security, and spiritual inspiration via employment, design aesthetics, access to nature, music, social interaction and the arts. To satisfy our definition of "new-green" a technology has to provide for one or more of these while having minimal or zero adverse environmental impact. Defining that in scientific terms comes next. 


\section{THE GREEN NANOTECHNOLOGY CHAIN; FROM SCIENCE TO PRODUCTS}

The aim here is to provide a rigorous basis for evaluating the "greenness" of a technology, first in terms of the underlying science, then in terms of potential for beneficial impact on both the environment and human well being. Such a framework is important given the proliferation of emerging products and opportunities in this field because we cannot afford in the short time needed for impact to have limited but growing financial and research resources squandered. The $R \& D$ and entrepreneurial processes inevitably involve some costly dead ends and failures, but with a more solid basis for evaluation of new ideas more productive investment decisions become possible along the development chain, and failures can embody more valuable lessons. Picking "winners" is never easy, but odds of success can always be improved.

A particular product may involve a development “chain” but the real value in more appropriate core science is in the multiplicity of such chains it can spin-off. As a well known example, basic quantum theory of chemical bonding and band structure led to semiconductors, which spun-off many simple optoelectronic and electrical devices, which led to the massive technological edifice behind nearly all $21^{\text {st }}$ century activity, and finally a transformation of human society which is ongoing. A better overall image is thus of a "tree" such as that whose components are projected onto figure 1. In this example optical and thermal science are the roots,

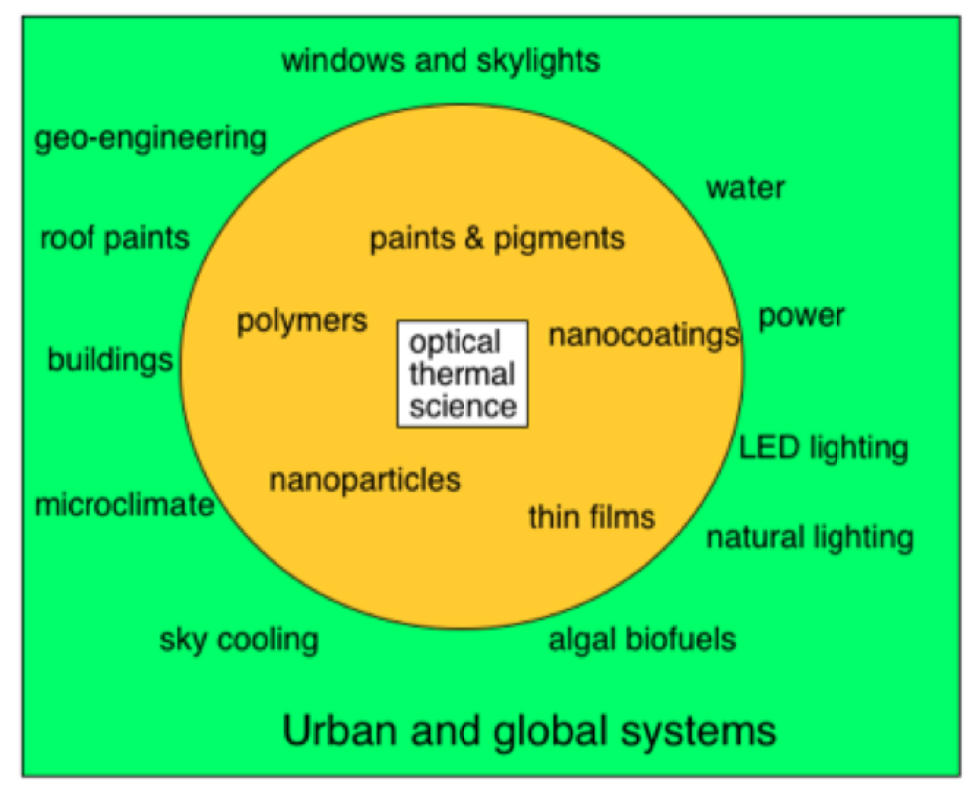

Figure 1. A green nanotechnology "tree", from basic science, through engineered materials, to technologies the materials can influence, and finally to their impact on global and local systems.

various special materials are the trunk, branches are numerous products, and leaves and fruit are the ensuing transformations in society and the global environment. The transformations underway now mean that the current young and future generations will experience a much different world to their immediate forbears. If we get this right it can be a much better world. "new-green science" involves many branches of physics, but thermal/optical are enough to illustrate our purposes.

Where does nanotechnology fit in? It is based on applications of a special large group of materials, some of which may look little changed in appearance from what they are replacing. This optical attribute of many nano-systems is not to be underrated. It facilitates retrofitting and allows savings gains without any unease for those that prefer a feeling of visual continuity. A thorough analysis of the core scientific philosophy from 
which green nanotechnology grows is to be found in the opening chapters of the recent book my Smith and Granqvist [2]. An outline follows. Our focus is on applications in the built environment, which is where the need for materials at commodity scale is largest and substantial energy savings and lifestyle gains are both possible. The best available green solutions at any time will include new and well-known microstructured and regular materials, but ultra-thin films and nanostructured materials are becoming more prevalent because they provide low cost ways of achieving desired functionalities over large areas. Special polymers and organic materials will also play an increasing role, especially in large area, affordable optical systems. Novel functional polymeric solids including polymer coatings are often nanostructured, for example when crosslinked, doped or imprinted. It is the link between nanostructure, response to the environment and efficient functionality that defines the science needed. Material responses must be tailored both to environmental energy flows and to human needs.

As a simplified generic example of how to build up from core science, take an opaque three layer material system interacting with the three dominant environmental incoming flows of energy (solar radiation, atmospheric thermal radiation, wind) along with subsequent flows as shown in figure 2.

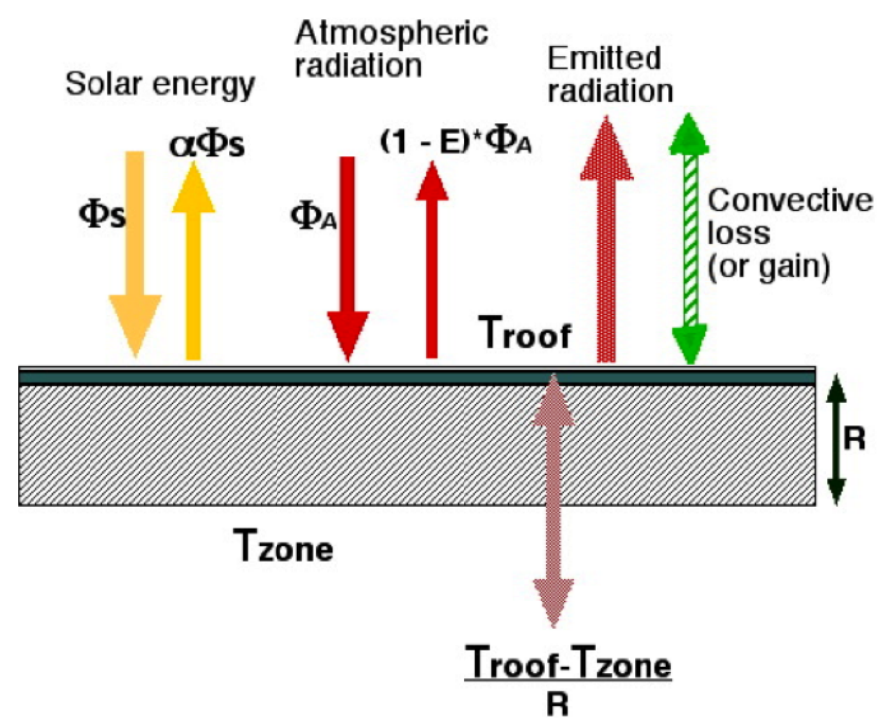

Figure 2. Incoming dominant environmental energy fluxes, resultant flows and resulting steady state temperatures. Three of the flows depend as shown on material properties; solar reflectance $\alpha$, thermal emittance E, and sub-roof insulation R-value, all of which can be controlled or improved with nano-engineering.

This could be a building roof where heat flows link to human comfort. It could be part of a solar thermal collector, where heat supplies a human need of hot water or electric power. The three materials are an outer coating, roof or collector heat exchange material, and sub-roof or sub-collector insulation. A common trap is to focus on the properties of one material component only, but the three material parameters together dictate net-heat flows (with the addition of internal fluid dynamics if the middle layer is a collector). An integrated approach is most important with large changes in incoming flows between night and day, and with seasonal variations [3]. They are each defined in terms of actual environmental flows at any instant and associated material spectral response [2], namely albedo $\alpha(\theta, \phi)$ [or solar reflectance $R_{\text {sol }}(\theta, \phi)$ ], which varies with incident direction $(\theta, \phi)$ and surface orientation, thermal emittance $\mathrm{E}$, and insulation R-value.

$1-\mathrm{A}_{\text {sol }}=\alpha$ with $\mathrm{A}_{\text {sol }}$ the solar absorptance. The energy balance in figure 2 can be represented mathematically at time $t$ as in equation (1) for unit area. 


$$
(1-\alpha) \Phi_{S}(t)+E \Phi_{A}\left(t, T_{A}, T_{\text {sky }}\right)-E \sigma T_{\text {roof }}^{4}-\Phi_{C}\left(T_{\text {roof }}, T_{A}, v_{w}\right)=\frac{1}{R}\left[T_{\text {roof }}-T_{\text {zone }}\right]
$$

with $\Phi_{\mathrm{s}}$ incident solar power, $\Phi_{\mathrm{A}}$ total down-welling atmospheric radiant power, $\mathrm{T}_{\mathrm{A}}$ local ambient temperature, $\Phi_{C}$ convective power exchange (which can be negative if roof temperature $T_{\text {roof }}<T_{A}$ which is often possible at night), $T_{\text {sky }}$ is effective sky temperature, $T_{\text {zone }}$ is interior temperature, $v_{w}$ is wind speed. The inwards flow through $\mathrm{R}$ can also be negative (ie outwards) if night sky cooling along with other factors such as internal loads and thermal storage lead to $\mathrm{T}_{\text {roof }}<\mathrm{T}_{\text {zone. }}$. If the roof material plus insulation is transmitting additional terms for transmitted radiation are needed. Managing $\Phi_{\mathrm{C}}$ may involve an additional outer transparent element not shown in figure 2. Its optical and radiative properties must also be carefully chosen.

Coated and bulk materials are often compared by their normal incidence value of $\alpha$, but computer simulation needs angular algorithms or data (for windows see the International Glazing Database [4]). Standard environmental incident spectra and surface orientation are often used, such as $\Phi_{\mathrm{S}}$ at normal incidence after passing though 1.5 air masses (AM1.5), $\Phi_{\mathrm{A}}$ when sample is normal to the zenith and the air is dry, and hemispherical emittance E. Total and instantaneous performance requires $\Phi_{\mathrm{S}}$ and $\Phi_{\mathrm{A}}$ to both vary over time to account for installed geometry, local weather, and normal environmental dynamics including night-day shifts in both radiant energies and air-flows. Finer details of the solar and atmospheric radiant spectra and their incident profile are often needed. The material then needs even finer tuning of spectral response, incidenceexit angle response, and scattering profile. Such tuning ability is a strength of nano-engineering.

Examples of desired optical combinations include

- daylight gain with good solar heat rejection

- color with high solar reflectance

- clear view plus useful daylight without glare or solar heat

- high reflectance of incoming thermal radiation except where the atmosphere is IR transparent

- $\quad$ strong insulating ability with high transmittance

- blocking solar radiation at directions of incidence high above the horizontal but transmitting for near normal and lower incident directions (for daylight and view).

- uniform lux distribution from a skylight or window despite seasonal and daily variations

- minimized localisation or maximized uniformity of daylight related lux levels

Dynamic control is useful and can be mechanical or intrinsic to materials. A response to the large time, direction and spectral dependent variations in external energy intensities needs a special category of materials in which thin films and nanostructures have growing roles. These are the chromogenic materials whose optical and/or thermal radiation responses can change with time, either in an auto-response to solar and thermal conditions or in response to sensor signals. Switching of optical responses is driven by changes in 
- temperature

- $\quad$ applied voltage

- UV radiation

- hydrogen gas

- daylight intensity thermochromic if spectral based, thermotropic if scattering based

electrochromic

photochromic

gasochromic

fluorescent (for enhanced visual contrast in bright sun)

Ideal spectral and angular dependent responses, plus examples of how nanostructures can get close to these ideals follow in section 3. It is interesting from a broader green technology perspective that many of the better chromogenic materials and related nanophotonic science have much in common with material developments in advanced batteries, supercapacitors and hydrogen storage materials [5, 2]. For example lithium ions intercalating or bonding into nanoporous oxide electrodes are used in electrochromics, select nickel and magnesium based alloys change optically as they absorb or lose hydrogen, doped $\mathrm{VO}_{2}$ thin films can be thermochromic with good visible transmittance [6], nanoparticles of material which undergo a metal insulator phase transition can modulate solar heat gains via on or off switching of an NIR localized surface plasmon resonance (without changes in visible transmittance)[7]. High performance transparent conducting electrodes are also needed in some of these and developments to get away from use of ITO, involve special nanomaterials including graphene and some special thin multi-layers.

Section 3 following focuses on energy efficiency. Energy supply derived using solar thermal or photovoltaics increasingly uses nanostructures in one or more components. Nanocomposites have become the material of choice in high quality spectrally selective solar absorbers, which have high $\mathrm{A}_{\text {sol }}$ and low E, including as used in the evacuated collector tube of large area parabolic trough electric power systems. These rely again on resonant absorption in the conducting component. A variety of nanostructures are being used in production and research level solar cells. Nanostructure in the transparent electrodes is available in mass production via for example pyrolytic deposition on float glass. They can achieve high conductivity and high transmittance combined plus enhanced light capture in the cell. If the cells use thin polycrystalline-Si this enables a single layer. Plasmonic nanoparticles displaced from the cell by a few nm thick passivation layer can also enhance photon coupling [8]. Related research developments include improvements in very thin (and non-ITO) transparent electrodes for inorganic and organic cells [9], enabling highly flexible plastic substrates, enhanced durability and enhanced charge coupling via reduced work function for charge transfer into devices. These developments carry over into light source developments mainly for LED's and OLED's, and also into all plastic or flexible general electronics. Low cost anti-reflection, including ability to maintain low reflectance over wide ranges of incidence, is of high interest for enhanced efficiency and amenable to various nanoporous structures and bio-mimickry.

\section{APPROACHING IDEAL OPTICAL RESPONSES WITH NANOSTRUCTURES}

Ideal spectral responses for opaque materials for use in solar thermal collectors and its exact opposite or spectral complement, which is ideal for cool roofs and some cool collector systems, appear in figure 3(a). The broken line in the visible zone is one of several variants for the cooling case in which color is added for aesthetics but there is retention of good but not as high solar reflectance as all NIR radiation is still reflected. An ideal transmittance spectrum for windows and skylights in warm and hot climates is in figure 3(b), with options for color and just how much daylight is admitted for glare control. The low transmittance spectral

zones are preferably achieved via high reflectance especially for Planck wavelengths, though a useful low 
cost alternative for the NIR band is absorption blocking resulting in slightly higher indirect solar heat gain via warmer glass.

(a)

(b)
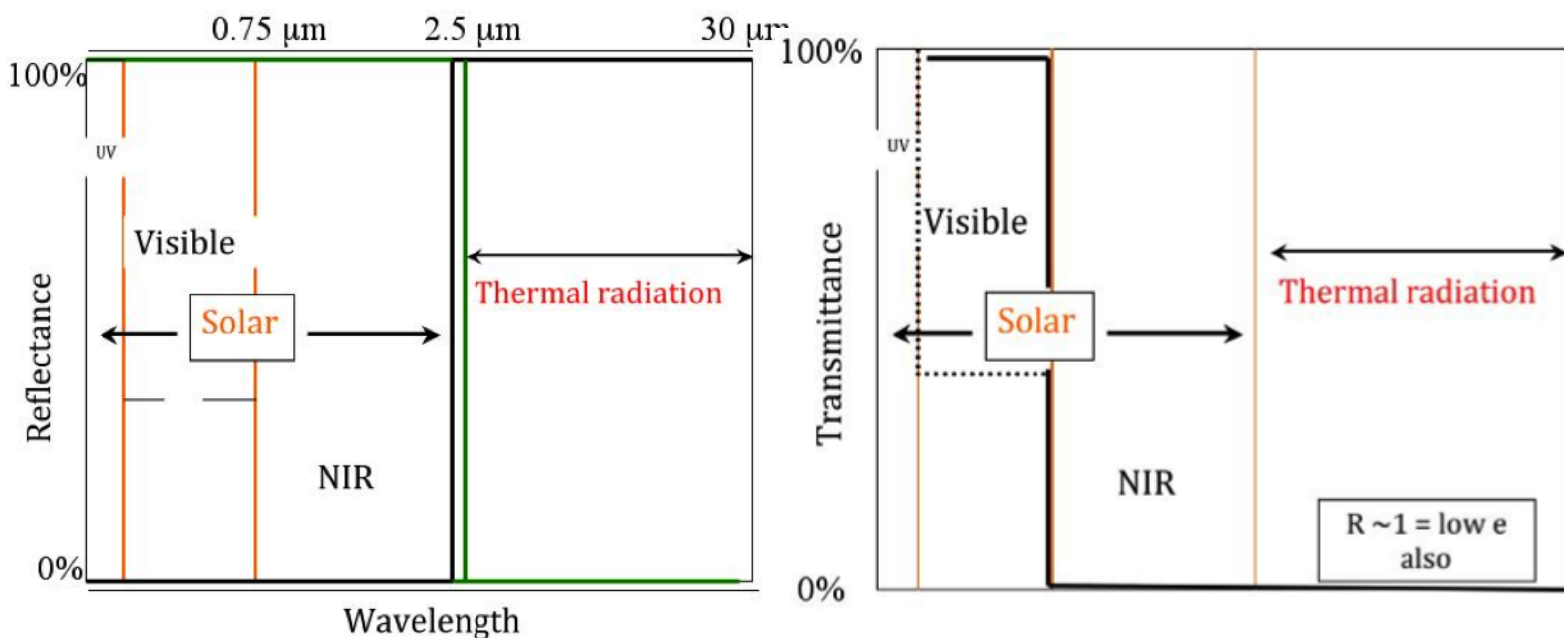

Ideal cool paint Ideal solar absorber

Figure 3. Three example spectral ideals (a) solar heat collectors, and cool roofs (b) windows in warm and hot climates, which admit daylight but minimize heat gain.

Figure 4 (a) shows a limiting practical example of fig 3(a) with color, namely a visibly black roof which stays cool by reflecting a lot of solar energy. Many other colors with high NIR reflectance and hence higher $\mathrm{R}_{\text {sol }}$ are available [10]. Such responses can be achieved using combinations of different nano- and micro-pigments and/or dyes, plus standard micro-particles and binders. Absorbing nano-pigments must not have high attenuation in the NIR. It is not often realised that high NIR reflectance is not always present after painting something white. That is some "whites" do not necessarily have high albedos. The best white paints can achieve solar reflectance around $90 \%$ to $93 \%$, the worst whitish paints only around $50 \%$ to 55\%. Figure 4(b) shows a practical example of a very high quality cermet solar absorber. It has three layers each around 70 to $80 \mathrm{~nm}$ thick. The base layer has medium ( 50\%) Ni metal content, the middle layer low Ni metal content ( $0.5 \%$ ) and the top layer is an insulator designed to allow more light to enter the absorbing segments.

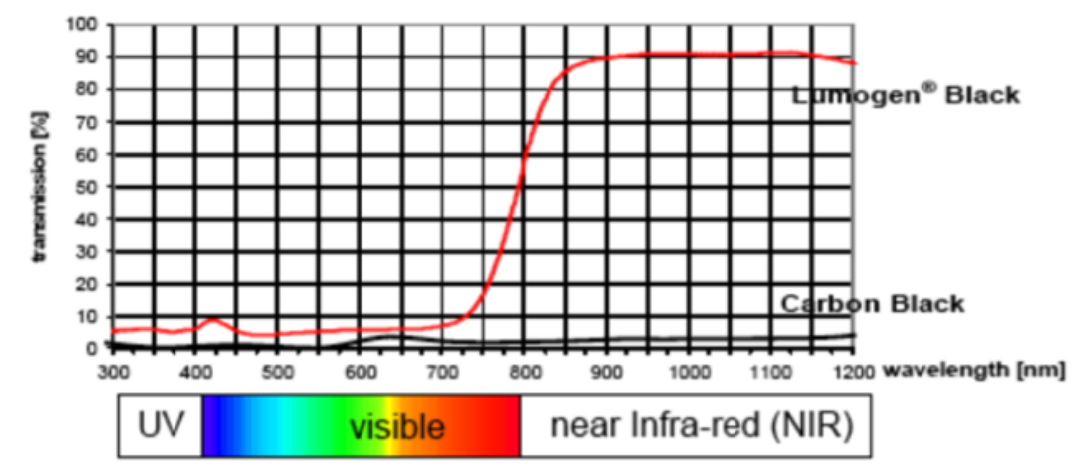

Figure 4(a). A black solar reflecting paint ([2] with permission, B. Schuler, BASF). 


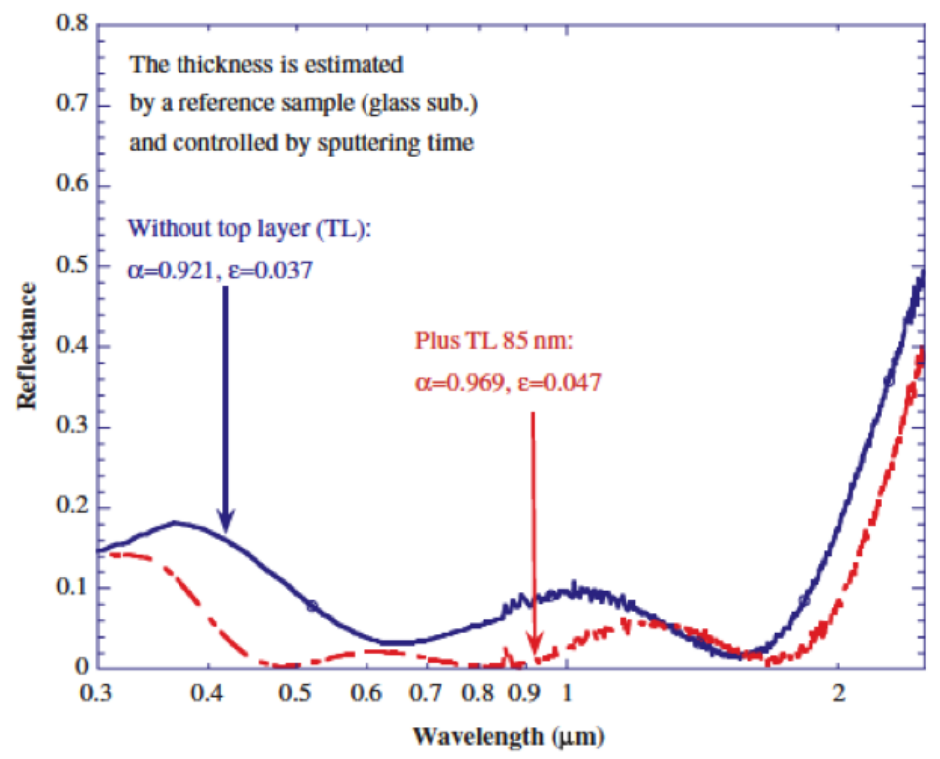

Figure 4(b). A near ideal solar absorber with $\mathrm{A}_{\text {sol }}=0.97$, and three nano-thin layers (red), two of which are nickel doped $\mathrm{NiO}$ at quite different concentration levels [2]. This film is on a metal substrate for high Planck radiation reflectance and low $\mathrm{E}=0.04$. The spectrum without the top layer is also shown. The top AR layer adds a useful 5\% to $\mathrm{A}_{\text {sol }}[11]$.

For achieving close to the ideal spectra shown in Figure 3(b) for solar control windows the established approach is multilayer thin film stack containing one or two very thin silver layers around $12 \mathrm{~nm}$ thick. Some of the insulating layers in the stack are even thinner. All layers are controlled to sub-nm accuracies in thickness and surface smoothness. Surface smoothness, plus internal and surface loss in nano-thin Ag is influenced by the choice of adjacent insulating layers and is important as nano-features in thin metal can lead to additional unwanted absorption via scattering and surface plasmons. This is one of the longest established examples of a mass produced building product involving precision nano-systems. It demonstrates that very large area production is feasible with over 60 million $\mathrm{m}^{2}$ per annum and growing. Example transmittance and reflectance spectra appear in figure 5. Visible transmittance is high, while NIR transmittance is low to reduce solar gain. In addition NIR reflectance is high so the window stays cooler, and Planck reflectance is also high leading to very low thermal emittance and excellent insulating ability.

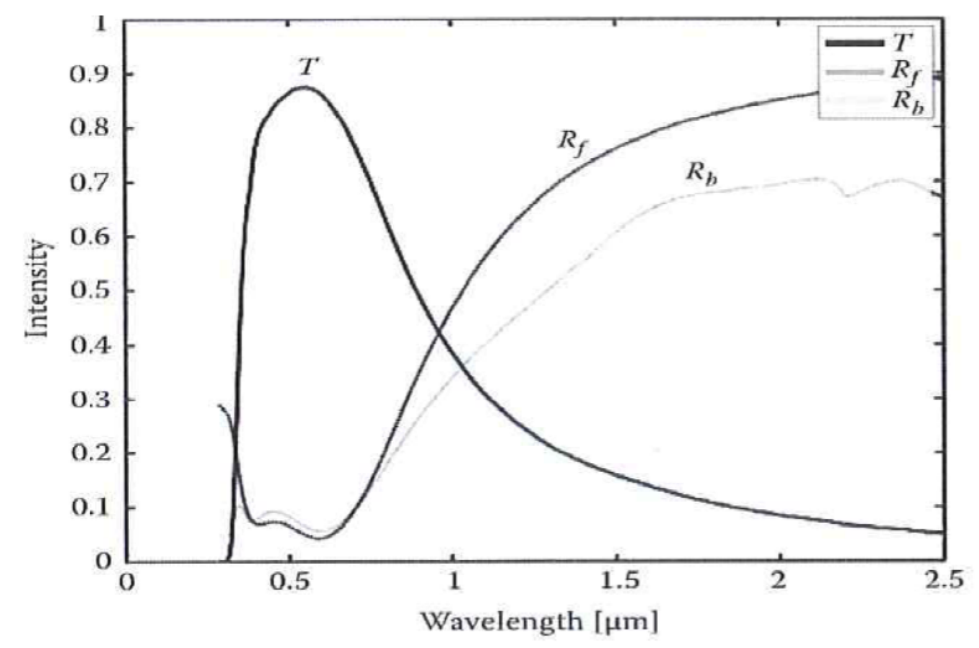

Figure 5. Transmittance and reflectance spectra for a solar control multilayer thin film on glass [2]. 
Doping of polymer layers with select plasmonic nanoparticles can produce similar transmittance spectra to that in figure 5 as long as their narrow absorption band is suitably located $[12,13]$. The same reflectance spectra is not achieved as these rely instead on blocking by absorption. Nanoparticle doping can however maintain a clear view as scattering is negligible if particles are small enough. Due to the strength of the surface plasmon resonance and its near ideal peak wavelength, very low concentrations will supply the desired spectral selectivity (for daylight with low heat), as shown in figure 6 for $\mathrm{LaB}_{6}$ nanoparticles in $0.7 \mathrm{~mm}$ thick PVB within a laminated glass system.

Plasmonic effects in thin film nanostructures can also be used to achieve angular selectivity [14, 15] provided the metal columns involved are oblique, which is achievable with Glancing Angle Deposition (GLAD). Again using nanostructures the view to the outside can be preserved and daylighting maintained. Other approaches to angular selectivity involve total internal reflection (TIR) from bulk or surface discontinuities in polymers. Though suited to glare and solar heat gain control and providing more uniform lighting response over a day and season in skylights, these usually suffer in normal windows from view distortion due to complex wave and scattering phenomena including the Goos-Hänchen shift near sharp structural discontinuities [16].

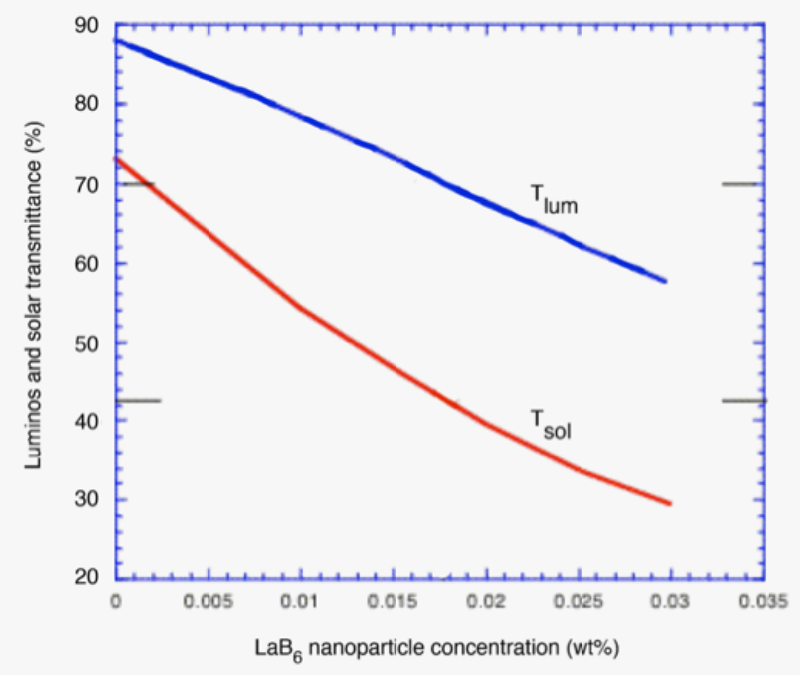

Figure 6. Luminous and solar transmittance with various low concentrations of $\mathrm{LaB}_{6}$ nanoparticles in $0.7 \mathrm{~mm}$ thick PVB between glass sheets.

\subsection{Efficient lighting, better quality daylighting and thermal insulation using nano-media.}

Quality lighting and energy efficient lighting are often considered separately but are inter-related. Light diffusion plays a key role in both, but the scattering materials and designs used must be carefully designed to achieve the desired goals. Quality involves up to four or five issues (i) sufficient lux for tasks (ii) low glare (iii) desired color rendering (iv) desired spatial distribution (v) aesthetic appeal. Spatial distribution is important but often neglected. The way our eyes and brains react together to widely varying light intensities makes us sensitive to large discontinuities in brightness levels. A reduced total lumen and lux level over a wide area can actually make a room appear brighter than a very high lumen level concentrated over a small fraction of the floor area. Energy efficiency results from reduced power use in lighting for which the three avenues are high efficacy lamps (high lumens/watt), high light output ratio (LOR) luminaires (lamp housing 
and light distribution systems), and maximizing use of daylight from windows, skylights and roof glazing. Supplying daylight with minimal solar heat was addressed in the previous section. It should also involve good thermal insulation via net U-value which has contributions from surface emittance E, conduction and convection. Reducing heat flow is specially important via roof glazing and skylights and can be achieved not only with multiple glazing but with special inorganic and polymer nanostructures that have both high transmittance and high $\mathrm{R}$ value where $\mathrm{R}=1 / \mathrm{U}$. Silica aerogel is an example and with excellent $\mathrm{R}$ at low thickness, has a high transmittance as seen in figure 7 [17]. Nanopolymers are less brittle, but aerogel is now practical if used in granule form for filling cavities in double-glazing or multi-wall polymer roofing,

Porous nanostructures in which the solid material is a poor thermal conductor make excellent insulators because in nano-pores convective flow cannot be established. It is this flow, not solid-state thermal conductivity, that dominates heat flow across traditional insulating slabs based often on fibre-glass and polystyrene. The nano-porous solid component is the residue in figure 8 . Total heat flow, and hence R-value at a given thickness, varies as a function of pore size to mean free path as exemplified in figure 8 [18].

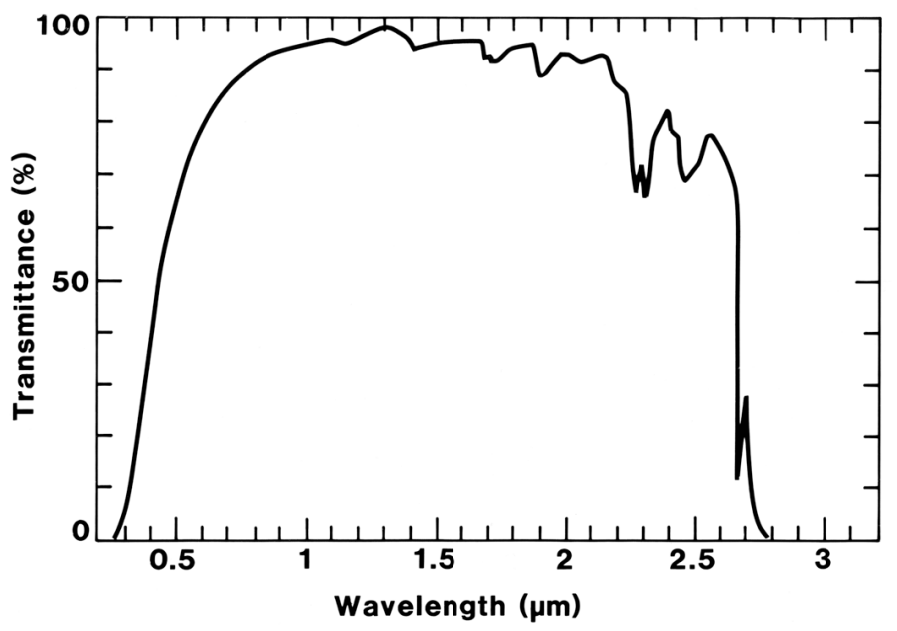

Figure 7 Spectral transmittance of a 4 mm thick layer of aerogel as used under or within roof glazing for insulation [17, 2].

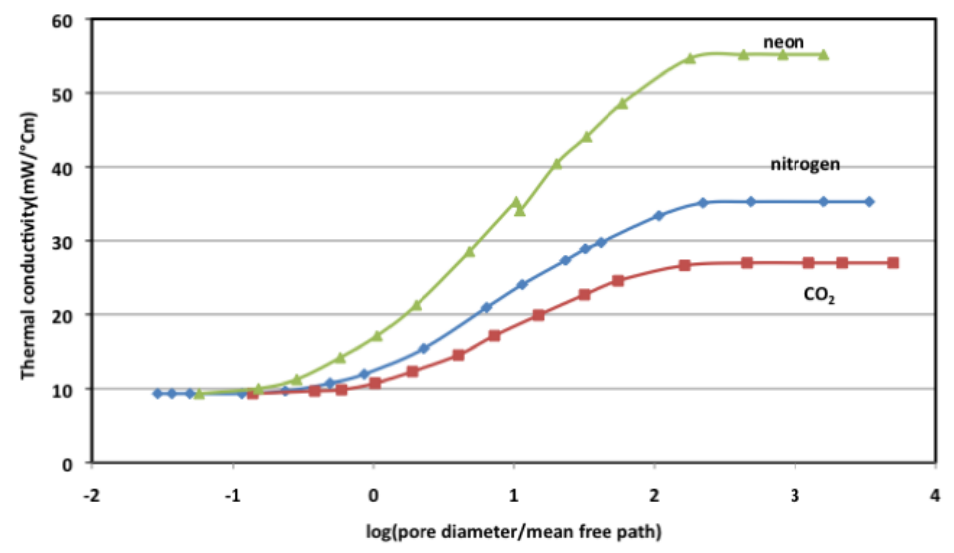

Figure 8 Impact of nanopore size relative to mean free path on thermal conductivity in a nanoporous solid containing gases of different molecular weight $[2,18]$. 
A goal of energy efficient diffusing materials for lighting fixtures and light distribution systems is high transmittance combined with low glare, evenly distributed lux and minimal color shift. A dominance of forward scattering across the whole visible spectrum is then desirable and is available in a well established class of doped polymers involving PMMA in PMMA with the dopant having a slightly different refractive index to the host PMMA via internal nanostructure with more cross-linking [19]. This also makes production easy since the dopants soften but don't melt in moulding, even though the host melts. A far from Lambertian output distribution may arise, which might be useful in itself. A wider light spread without loss in efficiency is also possible using ancillary design features, including lamp offset or multiple small lamps. Ideal skylight diffusing materials are different as they benefit from backscattering, which typically reduces solar heat gains by $\sim 50 \%$. Even though lumen input is lowered as well, we have found internal light distribution and average lux levels combined under clear skies represent much better overall lighting quality than non-diffusing skylights with their higher total visible transmittance, hence higher lumen input, for the same skylight area but less resulting spread.

\subsection{Nanostructures for infra-red spectral selectivity and night sky cooling}

Just as surface resonances from free electrons in conducting nanoparticles can be used for fine tuning at solar wavelengths, surface phonon resonances in select insulating nanoparticles can be used to fine tune infra-red absorption bands using nanoparticle doped polymers or as coatings on metal [20]. This resonance occurs where the smooth insulating material is a strong reflector due to having a negative dielectric constant (c.f. conducting reflectors). This is the Reststrahlen band. Combined mass of the cations and anions in each compound unit determine its resonant location. The ideal for night sky cooling to well below ambient is absorption and hence high emittance confined to wavelengths where the clear sky atmosphere is most transmissive which is between $7.9 \mu \mathrm{m}$ and $13 \mu \mathrm{m}$. Examples appear in figure 9 for $\mathrm{SiC}$ and $\mathrm{SiO}_{2}$ nanoparticles in PE foils. Temperatures and net heat pumping well below ambient can be achieved with such coatings in thin polymer layers on metal if set up under suitable IR transmitting covers (we have got to $15^{\circ} \mathrm{C}$ below coldest ambient in simple systems). This is because most incoming atmospheric radiation is reflected, while some radiation is still emitted but at wavelengths where there is little incoming atmospheric radiation. The special covers are needed to reduce inflow of heat from local air. Select polymers also provide useful spectrally selective radiation, though they can be improved with addition of nanoparticles. For many applications including open cool roofs, and roofs with special covers high emittance combined with high albedo as in figure 3(a) is still preferred. This is because cooling to a few degrees below the coldest ambient of the night is adequate and allows more heat to be pumped, and if desired for later use, "cold" to be stored.

The most important factor for all cool roofs is a high albedo. Considering figure 1 again, as albedo increases the sensitivity of heat gain and hence cooling load in homes to both emittance and sub-roof R-value decreases [3]. Hence the high cost of raising $\mathrm{R}$ to well above $\mathrm{R} \sim 1.63 \mathrm{Km}^{2} / \mathrm{W}$ (as found in $50 \mathrm{~mm}$ thick fibre glass) is not needed if albedo is high. The environmental and energy saving return on investment in coating to raise albedo is usually far better than raising $\mathrm{R}$. Raising $\mathrm{R}$ too much may even be deleterious for many nonresidential structures as these tend to have high internal loads. Then night sky cooling via high emittance can help a lot in overall cooling but is unable to do so if heat is trapped internally. An ideal practical combination is $\alpha>0.8, \mathrm{E}>0.9$. Intermediate $\mathrm{R}$ is preferred if winter heating is also an issue as long as albedo is high. Winter heating needs are much less sensitive to changes in roof albedo than summer cooling loads. Cool roofs have multiple important additional benefits, which are noted briefly next. 

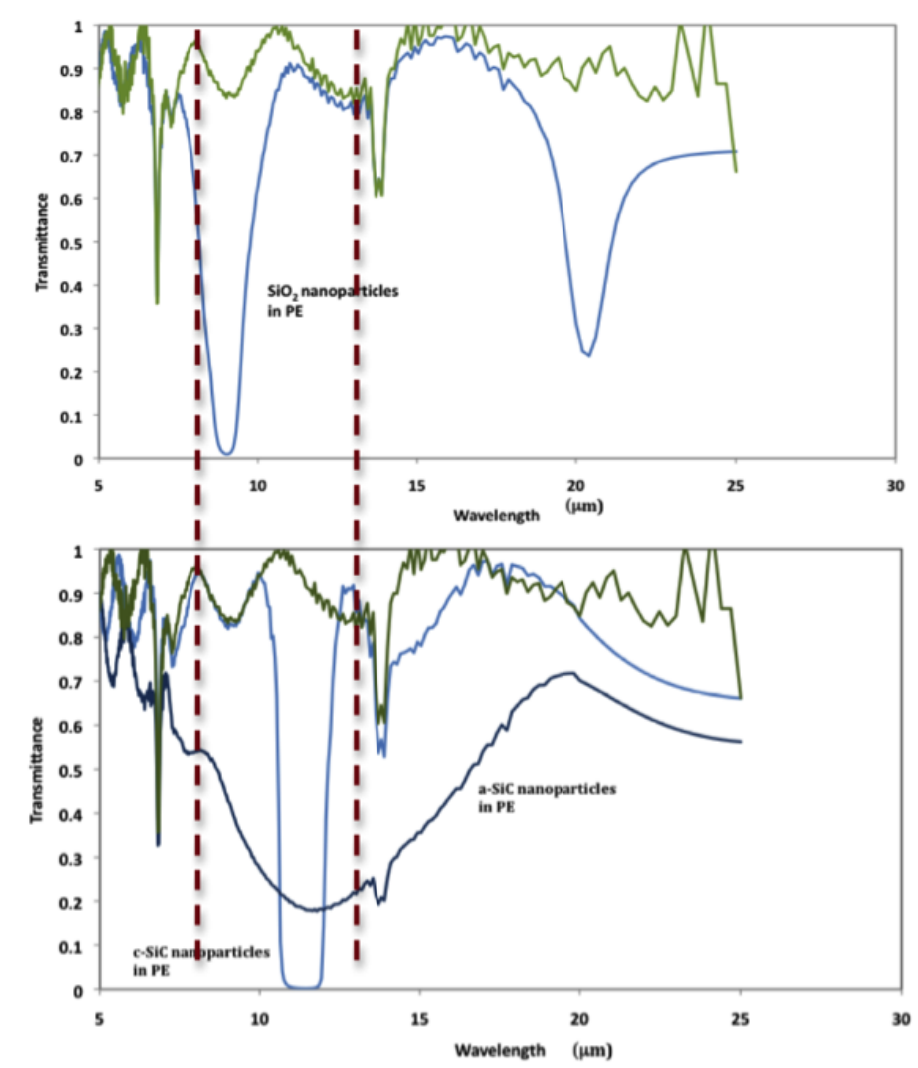

Figure 9. Surface phonon resonance absorption in $20 \mu \mathrm{m}$ thick $\mathrm{PE}$ foils doped respectively with $\mathrm{SiO}_{2}$ and $\mathrm{SiC}$ nanoparticles [20]. The second image shows the difference between c-SiC and amorphous SiC particles. The additional resonance in $\mathrm{SiO}_{2}$ near $20 \mu \mathrm{m}$ is where there is little incoming radiation as the relevant Planck radiation spectrum is weak and near flat by then.

\section{BROADER ISSUES AND FUTURE POSSIBILITIES}

Many issues beyond those discussed in the preceding section arise. Designing nano-materials to link environmental energy flows to human needs, and producing them in a laboratory is just the start. As with the sputtered solar control glazings and forward scattering polymers, focus ultimately has to be on those materials which can be fabricated at commodity scales. Affordability and functionality in paints, cool roofs, solar thermal and night "coolness" collectors, energy efficient windows and skylights, extruded and injection moulded composite polymers, organic and thin film solar cells, OLED's, thermal insulators, nanoporous structural materials and membranes, and many more such products will be enhanced in performance and cost by nanostructures. Controlled engineering of nanomaterials at these areas and volumes has unique challenges, which should be kept in mind. Structures and hence optical response will often change in manufacture, sometimes beneficially. Energy and temperatures needed in manufacture is a consideration. For example flexible nano-thin transparent conductors on plastic using alternatives to ITO are ideally deposited at substrate temperatures of $50^{\circ} \mathrm{C}$ or lower to avoid damage to the base polymer. Nanoparticles can change shape, cluster or align during processing and host materials properties can also change. Final optical responses then shift, in some cases dramatically.

Systems analysis under actual local weather and atmospheric conditions and over 24 hours instead of just the daytime can change one's perspective on the optimum properties for each component but especially on how 
to achieve maximum environmental impact per dollar invested. Cool roofs were a good example, where we found that having a very high sub-roof R-value has little additional benefit if albedo is high, but adds a lot of extra cost. If thermal emittance is also high raising $\mathrm{R}$ can actually lower performance especially if internal loads are high and/or clear dry skies are usually present. It is also worth broadening system considerations even further. For example if PV systems are mounted on a cool roof as opposed to on one with mid to low albedo, useful electric output gains can arise with little or no additional cost. Another example is impact on cooling COP's, especially of roof mounted air conditioning units (common in large buildings), but also even if near cooler walls. COP's can be a lot higher if intake air temperatures drop. Air near medium to low albedo roofs can reach around $45^{\circ} \mathrm{C}$ to $60^{\circ} \mathrm{C}$ while that near a cool roof may stay a few degrees above ambient. A COP rise from around 2.0 to around 3.0 to 4.0 is then feasible [21] with R22 and R410A cooling systems. Resultant savings in power use and carbon emissions are large. Additional benefits should flow from air exchange using cooler local air. For both PV and COP cases night sky cooling adds additional performance over the ensuing day.

The right materials combinations also provide ancillary benefits beyond the single building analysis. Peak demand reductions are one bonus if enough cool roofs exist. Meeting peak demand is demanding on grid capital investment, power sources and chiller capacity. Avoiding overheating discomfort on a handful of worst days each year is the core problem. Its amelioration with better building design is not difficult, from cool roofs and controlled night cooling with sky radiation, and letting in local cool air when available. Peak (thermal) cooling load contributions if albedo rises by just 0.2 with $\mathrm{R}=1.63 \mathrm{Km} / \mathrm{W}$ reduce by $1.5 \mathrm{~kW}$ to $2 \mathrm{~kW}$ per $100 \mathrm{~m}^{2}$ of roof [3] and even with $\mathrm{R}=3.06$ (double thickness) $\sim 1 \mathrm{~kW}$ per $100 \mathrm{~m}^{2}$ of thermal gains at peak are avoidable. With larger shifts in albedo, which are often feasible, even larger savings are possible.

Future studies need to look more carefully at the impact of cool roofs on local microclimates, and by extension if there are enough such roofs, whole precincts can become cooler. Enhanced night sky cooling can add to this impact as cold sub-ambient air flows off sky-cooled roofs. Combining these with local green shaded areas can make urban outdoor precinct conditions more pleasant and further reduce overall cooling demand.

Many future nano-developments may add to those outlined above [2]. Advances in thermoelectricity to enable more power from power station waste heat, car exhausts and solar heat, plus compact electronic cooling, holds much promise using nanostructures [22]. It has however formidable manufacturing challenges. Durable construction materials embodying nanostructures, especially using complex pores for strength and light weight, are needed. Adding intrinsic sensors and dynamic optical properties may change the way urban areas look and behave, with major environmental benefits. Water, humidity and condensation management will develop further with nano-systems.

A world based on "new-green" technology, with its large nanotechnology component, is an enticing prospect. Attaining such a world and then nurturing it must be one of today's core challenges.

\section{ACKNOWLEDGEMENTS}

Many colleagues and students have contributed to the ideas and science outlined above. Special mention goes to Angus Gentle, Claes Granqvist, Jim Franklin, Alan Earp, Gunnar Niklasson, Steve Lynch and Geoff McCredie, plus various staff at LBL in California, and ISE in Freiburg Germany. Support over many years from government and industry is gratefully acknowledged, including recent ARC Discovery and CSIRO Grants for cooling studies. 


\section{REFERENCES}

[1] I. Edmonds and G.B. Smith, "Surface reflectance and conversion efficiency dependence of technologies for mitigating global warming”, Renewable Energy 36 (2011) 1343-1351.

[2] G.B. Smith and C.G. Granqvist, [Green Nanotechnology solutions for sustainability and energy in the built environment] (CRC Press, Taylor and Francis, Boca Raton, FL USA , 2010).

[3] A.R. Gentle, J.L.C. Aguilar and G.B. Smith, "Optimized cool roofs: integrating albedo and thermal emittance with R-Value”, in press Solar Energy Materials and Solar Cells (2011).

[4] International Glazing Database (2009) Lawrence Berkeley National Laboratory, http://windows/lbl.gov/software/window.html

[5] A.S. Arico, P. Bruce, B. Scrosati, J.-M. Tarascon W. van Schalkwijk . "Nanostructured materials for advanced energy conversion and storage devices”, Nature Materials 4 (2005) 366-377.

[6] N.R. Mlyuka, G.A. Niklasson and C.G. Granqvist, "Mg doping of thermochromic $\mathrm{VO}_{2}$ films enhances the optical transmittance and decreases the metal-insulator transition temperature”, Appl. Phys. Lett. 95 (2009) 171909 1-3.

[7] S. Y. Li, G. A. Niklasson, and C. G. Granqvist, "Nanothermochromics: Calculations for VO2 nanoparticles in dielectric hosts show much improved luminous transmittance and solar energy transmittance modulation,” J. Appl. Phys. 108 (2010) 063525.

[8] K.R. Catchpole, and A. Polman, "Plasmonic solar cells”, Optics Express 16 (2008) 21793-21800.

[9] C. Guillen and J. Herrero, "Transparent electrodes based on metal and metal oxide stacked layers grown at room temperature on polymer substrate”, Phys. Stat. Solidi A 207 (2010) 1563-1567.

[10] A. Synnefa, M. Santamouris, K. Apostolakis, “On the development, optical properties and thermal performance of cool colored coatings for the urban environment”, Solar Energy 81 (2007) 488-497.

[11] S. Zhou and E. Wäckelgård, "Optimization of solar absorbing three-layer coatings”, Solar Energy Materials \& Solar Cells 90 (2006) 243-261, doi:10.1016/j.solmat.2005.03.009

[12] S. Schlem and G. B. Smith, "Dilute LaB6 nanoparticles in polymer as optimised clear solar control glazing”, Appl. Phys. Lett. 82 (2003) 4346-4348.

[13] G.B. Smith, C.A. Deller, P.D. Swift, A.Gentle, P.D. Garrett and W.K.Fisher, "Nanoparticle doped polymer foils for use in solar control glazing”, Journal of Nanoparticle Research 4/1-2, (2002) 157 -165.

[14] G.B. Smith, S. Dligatch, R. Sullivan and M.G. Hutchins, “Thin Film Angular Selective Glazing, A Review”, Solar Energy 62 (1998) 229 - 244.

[15] G.W. Mbise, D. LeBellac, G.A. Niklasson and C.G. Granqvist, “Angular selective window coatings: theory and experiments”, J. Phys. D; Appl. Phys. 30 (1997) 2103-2122.

[16] G. Walze, A. Gombert, P. Nitz and B. Bläsi, "Rigorous validation of the lateral Goos-Hanchen shift in microstructured sun shading systems”, Proc. SPIE 6197Z 1-9 (2006)

[17] C.G. Granqvist, pgs 106-167 in “Materials Science for Solar Energy Conversion Systems” (edited by C.G. Granqvist, Pergamon, Oxford UK 1991).

[18] Derived by the author using data in J.C. Harper and A.F. El Sahrigi, “Thermal conductivity of gas filled porous solids”, Ind. Engr. Chem. Fundamentals 3 (1964) 318-324.

[19] G.B. Smith, J. Jonsson and J. Franklin "Spectral global and diffuse properties of high performance translucent polymer sheets for energy efficient lighting and skylights”, Applied Optics 42 (2003) 39813991.

[20] A.R. Gentle and G.B. Smith, "Radiative heat pumping from the earth using surface phonon resonant nanoparticles”, NanoLetters 10 (2010) 373-379.

[21] W. Vance Payne and Piotr A. Domanski, "A Comparison of an R22 and an R410A Air Conditioner Operating at High Ambient Temperatures, R2-1”, Proceedings International Refrigeration and Air Conditioning Conference, July 16-19, 2002, West Lafayette, IN.

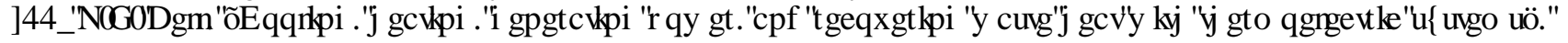

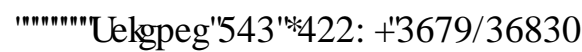

